\title{
STORIOGRAFIA CROCIANA E STORIA DELLE IDEE: L'ADAMO ED EVA DI ANTONELLO GERBI ${ }^{1}$
}

\section{Enrico Isacco Rambaldi ${ }^{2}$}

\begin{abstract}
Invaghitosi d'una giovane maritata, un frate le si dichiarò per lettera, lodandone le divine bellezze. "La donna, ancora che onestissima fosse, leggendo, tutta godeva per lo sentire le sue bellezze sí altamente lodare, sí come colei che aveva col peccato originale insiemi contratta la innata passione, che hanno già tutto lo resto del sesso femmineo". Il novellino ${ }^{3}$
\end{abstract}

RIASSUNTO: Antonello Gerbi (1904-1976), storico della filosofia e del pensiero politico, fu molto vicino a Benedetto Croce ed ebbe rapporti con Arthur Lovejoy. Impostato secondo il modello storiografico crociano, il suo libro Il peccato di Adamo ed Eva espone la storia delle concezioni del Peccato originale come peccato carnale dal II al XIX secolo. Per la vastità delle fonti prese in esame (filosofi, teologi, poeti, artisti ...) e per il rigore col quale unifica la ricerca attorno al tema centrale della carnalità della Caduta, il libro presenta interessanti affinità con la storia delle idee e testimonia della versatilità del metodo storiografico di Croce, aperto a integrarsi con quello di Lovejoy.

PAROLE CHIAVE: Peccato originale. Croce. Lovejoy.

${ }^{1}$ I riferimenti bibliografici sono ristretti alle sole opere indispensabili per suffragare le affermazioni critiche; i titoli tra [parentesi quadre] apparvero anonimi; le citazioni sono tra «caporali»; gli "apici alti" tra caporali segnalano citazioni fatte dall'autore citato; per le citazioni dall'Adamo ed Eva metto tra parentesi (tonda) solo il nr. di pagina; le parentesi [quadre] interne alle citazioni denotano miei interventi nei testi citati; segnalo le mie corsivazioni e i miei grassetti; emendo tacitamente ortografia e punteggiatura di autori del Novecento, mentre trascrivo in modo conservativo le citazioni di autori anteriori; poiché questo contributo viene pubblicato in una rivista non italiana, ritengo inutile tradurre in italiano le citazioni in inglese e francese; brevi e semplici le citazioni latine; talvolta, dove mi è parso profittevole per concisione, chiarezza e semplicità, cito direttamente dagli autori (Rousseau, Kant, Hegel, Francesco di Sales, Bergson, Erasmo).

${ }^{2}$ Nato a Milano nel 1936. Allievo di Mario Dal Pra, ha studiato anche in Germania, Svizzera, Francia e Regno Unito. È stato professore ordinario di Storia della filosofia e di Filosofia morale nell'Università di Milano. Si è occupato di storia della geometria nel Cinquecento, di storia della filosofia tedesca dell'Ottocento e del Novecento italiano. Dirige la Rivista di storia della filosofia. enrico.rambaldi@unimi.it

${ }^{3}$ Con qualche libertà da MASUCCIO, 1940, p. 10. 


\section{Premessa}

Ristampato nel 2011, a quasi ottant'anni dalla prima uscita (1933) e a trentacinque dalla morte dell'Autore, Il peccato di Adamo ed Eva di Antonello Gerbi (GERBI A., 2011) conserva intatta la freschezza di quando, per il vasto spettro di fonti filosofiche, religiose, teologiche e letterarie e per la ricca ricerca iconografica, segnò una novità nella storiografia filosofica italiana. Tra le singolarità del libro c'è un'affinità con la storia delle idee, e ciò lo rende esemplare per mettere a fuoco se, ed eventualmente in che misura, l'indirizzo storiografico promosso da Lovejoy, che si diffuse nel nostro secondo Novecento con la ventata di novità giunte in Italia nel dopoguerra, abbia davvero comportato una cesura radicale con la storiografia filosofica precedente e con l'anteriore rinnovamento attuato da Croce e Gentile (ma, per la storiografia, soprattutto da Croce), quando smantellarono il positivismo e fecero di Hegel un interlocutore non di scuola, ma vivo della nostra cultura.

Che questa cesura ci sia stata sembra recisamente affermarlo un esponente di spicco della rinnovata storiografia filosofica italiana, Paolo Rossi, laddove scrive che «[...] alla metà degli anni Trenta, quando Lovejoy pubblicava $\mathrm{La}$ Grande Catena dell'Essere, la storia della filosofia veniva presentata in Italia come un processo omogeneo, coerente e unitario, dotato di un carattere di continuità e svolgentesi secondo le leggi di un processo necessario»; e prosegue osservando che queste riduzioni «[...] della storia della filosofia a una sorta di atemporale esplorazione geografica delle regioni dello Spirito, della storia della letteratura a una distinzione tra poesia e non-poesia, non offrivano molto spazio ad una considerazione positiva dell'opera di Lovejoy» (ROSSI, 1982, p. 11). In questo giudizio, che con l'“atemporalità" delle categorie dello Spirito allude a Gentile $\mathrm{e}^{4}$ con la distinzione tra "poesia e non poesia" a Croce, Rossi, ch'ebbe un interesse vivissimo per Arthur Lovejoy e la storia delle idee, sembra di primo acchito affermare che nel dopoguerra quella cesura fosse inevitabile, e ch'egli stesso volesse contribuire ad allargarla. Ma Rossi era studioso troppo ricco e complesso per strappare da sé quelli che, anche tramite il suo maestro Eugenio Garin, erano stati gli interlocutori della sua prima, appassionata formazione ${ }^{5}$. Altrove, infatti,

\footnotetext{
${ }^{4}$ Altrove Rossi scrive che «[...] quando uscì negli Stati Uniti The Great Chain of Being (nel 1936), né gli storici della filosofia (impegnati a individuare "superamenti") né gli storici della letteratura (impegnati a distinguere poesia da non poesia) dedicarono una qualche attenzione a quel grande libro" (citato da RONCHETTI, 2002, p. 126).

${ }^{5} \mathrm{Si}$ veda quanto scrive Ferdinando Abbri sullo «studio accurato» e le «letture appassionate e intensissime» di Rossi negli anni a cavallo della laurea (1946); tra gli autori letti, oltre naturalmente a Croce e Gentile, anche Guido De Ruggiero e Guido Calogero (ABBRI, 2014, p. 336-337).
} 
ricorda che Gentile fu storico anche molto acuto e attento, e che nelle sue "[...] numerose ricerche di carattere particolare» la tendenza a presentare la storia del pensiero come un tutto omogeneo, che attraverso successivi "[...] superamenti" si svolge necessariamente verso l'immanentismo assoluto, è «[...] spesso frenata da una salda passione di storico, da una attenta cura del documento e della interpretazione precisa» (ROSSI, 1969, p. 36-37). Riguardo alla storiografia di Croce, poi, conservo vivissimo ricordo di Rossi giovane professore incaricato di Filosofia della storia nell'Università di Milano sul finire degli anni Cinquanta, che nelle lezioni illustrava gli aspetti analitici e determinati dell'opera storiografica di Croce e il principio che «[...] "l'attenzione dello studioso e del critico o dello storico della filosofia, deve riportarsi sempre sui particolari problemi che i filosofi propongono o risolvono o cercano di proporre e di risolvere" ", e che mostrava come, in stretta relazione col suo antifascismo, Croce avesse impresso al concetto di "contemporaneità della storia" (sul quale più avanti anche qui si tornerà) una forte tensione verso la realtà concreta, rendendolo atto «[...] ad agire, nella cultura italiana, come uno strumento da impiegarsi nella ricerca storiografica» (ivi, p. 26, 27).

La conoscenza esauriente della natura del trapasso che tra primo e secondo Novecento conobbe la cultura umanistica italiana può aversi solo con un vasto esame di quegli studiosi che, nati a cavallo tra XIX e XX secolo - Devoto, Dionisotti, Contini, Fubini, Chabod, Russo, Santoli, Gerbi... -, lavorarono nel solco dell'opera di Croce, ma con un'innovativa continuità

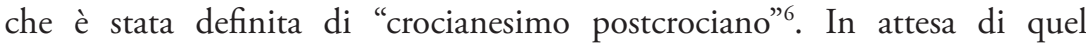
quadro completo, l'affinità "spontanea" che nell'Adamo ed Eva uno storico profondamente crociano come Gerbi manifesta con la storiografia delle idee è significativa testimonianza che se l'influenza di Croce "storico" e "critico" è sopravvissuta per non meno di un trentennio al periodo dell'egemonia neoidealistica, e se, tramontata quell'influenza diretta, si è però ravvivato l'interesse degli studiosi per l'opera sua, ciò è accaduto perché, altrimenti da quello di Gentile, il pensiero di Croce era "compatibile” con l'apertura a nuovi indirizzi critici e storiografici, coi quali si mostrò suscettibile di reciprochi arricchimenti e integrazioni; ad es. con la critica stilistica di Leo Spitzer, come mostra l'opera di Mario Fubini, o, appunto, con la storiografia di Arthur Lovejoy, come mostra quella di Antonello Gerbi.

È sotto quest'angolo di visuale, per il quale il libro è esemplare, che qui esamino le affinità tra l'Adamo ed Eva e la storia delle idee.

${ }^{6}$ L'espressione è in CONTINI, 1972, p. 67. 


\title{
2. Contenuto dell'Adamo ed EVA
}

Il libro narra la storia di due millenni d'interpretazioni carnali della Caduta, cioè che «[...] il frutto proibito da Dio ad Adamo [fosse] stato il godimento di Eva» (p. 15); interpretazioni che Gerbi, con riferimento al De Peccato Originali (1679) del libertino olandese Adriaan Beverland, raccoglie sotto la definizione comune di "ipotesi di Beverland", e delle quali esamina lo svolgimento con un'impostazione storiografica che ha le caratteristiche d'una morfologia fenomenologica connessa alla storia del pensiero umano.

Capostipite dell'interpretazione carnale è Filone Ebreo, che contamina le tradizioni mosaica, platonica e neoplatonica e ravvisa nella narrazione di Genesi non "[...] "finzioni mitiche, come quelle di cui si dilettano poeti e sofisti, bensì [...] modi che si richiamano all'interpretazione allegorica, consistente in spiegazioni che si imperniano sui significati riposti”» (p. 22). La Caduta diviene un'allegoria simile a quella del Simposio di Platone: maschio e femmina sono "[...] "le due parti divise di un unico essere dimezzato"», che l'amore «[...] "riunisce e riporta alla fusione" »; dal reciproco "[...] "desiderio di [Adamo ed Eva ad] unirsi”" scaturì "[...] "il piacere fisico, che è radice prima di iniquità e di prevaricazione” " (p. 22). Le diverse formulazioni, di varia origine confessionale (ebraiche, cristiane, manichee ...), della Caduta carnale vengono esposte da Gerbi in ordine storico-cronologico: dalle allegorie di Filone e da Clemente si passa alla Patristica, alle eresie d'impronta manichea tardoantiche e medievali e alla Scolastica, nel cui ambito Tommaso distingue tra l'aspetto materialiter carnale e quello formaliter spirituale della Caduta, relegando in secondo piano la peccaminosità sessuale; distinzione accolta da Dante, che fa dire ad Adamo

\author{
Or, figliuol mio, non il gustar del legno \\ Fu per sé la cagion di tanto essilio, \\ Ma solamente il trapassar del segno. (Par., XXVI, 115-117).
}

Per suffragare la tesi che nell'Umanesimo, nel Rinascimento e nella Riforma l'interpretazione carnale divenisse vieppiù realistica, Gerbi dota l'Adamo ed Eva di un corredo iconografico, che tra altri comprende un dipinto del 1517 di Hans Holbein il Giovane, nel quale Adamo ed Eva appaiono «[...] realistici e volgari, malinconici e vogliosi, veri archetipi d'una delusa e dolente umanità» (p. 110), e un'incisione di Jan Gossaert, dove "Adamo, in atto di desiderio, porta la sua gamba sinistra sopra la destra divaricata di 
Eva, la quale scopre il grembo e si protende quasi al bacio» (p. 112) ${ }^{7}$ Nel Seicento, in Paradise Lost Milton riscatta la peccaminosità della Caduta, che interpreta insieme come un male e come un bene, in quanto anche espressione della dignità e della libertà umane e premessa dell'Incarnazione. Sempre nel Seicento, il Peccato viene descritto da Beverland in modo "[...] beffardo, sacrilego, ghignante» (p. 132) come effetto di «[...] "coëundi pruritus"» (p. 13), e reso innocente dal venir equiparato all'accoppiamento tra due mammiferi del regno animale. La secolarizzazione procede nel Settecento; la Professione di fede del Vicario Savoiardo, ad es., trasforma le parole di Satana, "[...] eritis sicut dii, scientes bonum et malum» (Genesi, III, 5), in attribuiti positivi della coscienza: «Conscience, conscience! instinct divin [...] juge infaillible du bien et du mal, qui rends l'homme semblable à Dieu» (ROUSSEAU, 1969, p. 600-601). La critica profana della narrazione biblica si compie nella filosofia classica tedesca. In Kant, l'uscita dell'uomo «[...] da un giardino che lo nutriva senza lavoro» segna il passaggio «[...] dallo stato di bruto all'umanità [... ] allo stato di libertà» (KANT, 1965, p. 201, 202). Hegel spiega che la perdita del Paradiso «[...] per aver mangiato [il frutto] dell'albero della conoscenza del bene e del male» esprime la verità filosofica che «la conoscenza è il peccato originale» che fa uscire l'uomo dall'immediatezza naturale; "[...] perciò il peccato originale è il mito eterno dell'uomo, è il peccato tramite il quale l'uomo si fa uomo", mentre "lo stato d'innocenza, questa condizione paradisiaca, è lo stato animale. Il paradiso è un parco, dove possono rimanere solo gli animali, non già l'uomo» (HEGEL, 2003, p. 267).

La trattazione di Gerbi si conclude con Kierkegaard, Schopenhauer e Baudelaire. Kierkegaard ritiene che l'angoscia, caratteristica peculiare della coscienza umana, sia insieme frutto della "[...] sensualità turbata dalla coscienza del peccato» (p. 184) e fondamento della storia umana: gli angeli, che non conoscono sessualità né angoscia, non hanno storia. Per Schopenhauer, la sessualità è strumento della Volontà, che nel mito del Peccato originale celebra l'eterna vittoria sull'uomo, incatenato alla fatale e ripetitiva soddisfazione del piacere sessuale. Baudelaire riabilita Satana, che spingendo Adamo ed Eva alla sessualità insegnò loro «[...] "par l'amour le goût du Paradis"» (p. 192).

\footnotetext{
${ }^{7}$ Le immagini delle due opere sono visibili nei siti del "Kunstmuseum" di Basilea e dell'"Albertina Museum" di Vienna.
} 


\section{IL PECCATO DI ADAMO ED EVA E LA STORIA DELLE IDEE.}

Pur nella larga varietà di fonti religiose, teologiche, letterarie e iconografiche, l'Adamo ed Eva resta in primo luogo un libro di storiografia filosofica. Lo conferma la tavola tracciata da Gerbi per illustrare i centri nodali dai quali s'irradia la ricerca (figura 1$)^{8}$; vi figurano esponenti della vita religiosa (Ambrogio, Agostino, Lutero ...), pittori (Luca da Leida, Hugo van der Goes, Jan Gossaert ...), poeti (Novalis, Baudelaire ...); ma le tre direttrici verticali, ordinate lungo un asse cronologico che va dal II al XIX secolo, elencano soprattutto protagonisti della storia del pensiero: le interpretazioni che di Platone vennero proposte nei primi secoli dell'era volgare, Filone, Clemente, Origene, i neoplatonici, Agostino, Maimonide, Hamann, Herder, Kant, Schelling, Hegel, Schopenhauer, Hartmann, oltre a movimenti di pensiero (come quello libertino) e autori (Agrippa, Paracelso, Fludd ...) che solo più tardi entreranno a pieno titolo nella storiografia filosofica italiana.

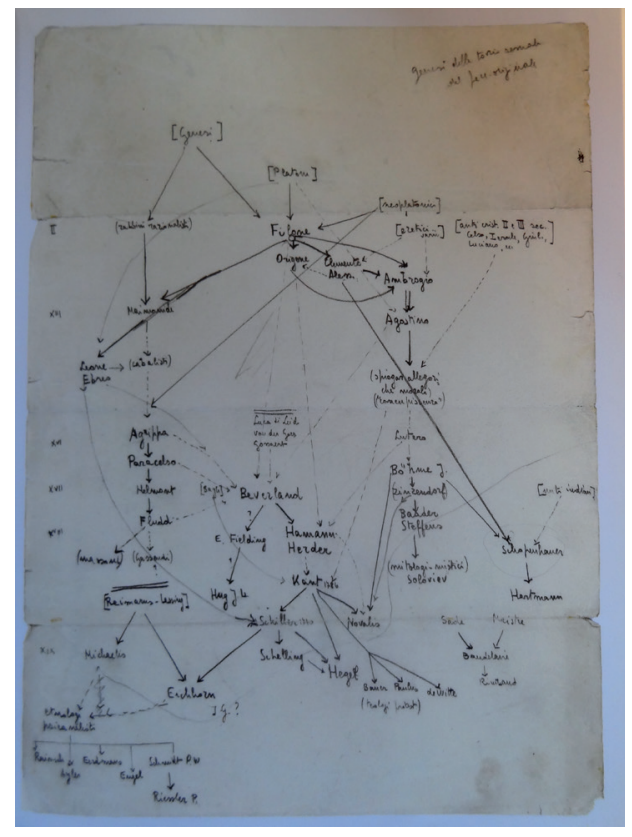

${ }^{8}$ La tavola riepilogativa «Genesi della teoria sessuale del pecc. originale» è riprodotta in PINOMONTANARI, 2007, p. 41. Non è datata, ma pare anteriore alla definitiva stesura dell'Adamo ed Eva, mancando autori che nel libro sono trattati (ad es. Tommaso, Rousseau, Kierkegaard, Holbein il Giovane); ne menziona però altri che nel libro non vengono ricordati (Rimbaud). Ringrazio vivamente Sandro Gerbi per avermi consentito di riprodurla qui. 
All'epoca, questa varietà rendeva l'Adamo ed Eva un'opera singolare, che può accostarsi a La carne, la morte e il diavolo nella letteratura romantica (citato nell'Adamo ed Eva) di Mario Praz; studioso che Gerbi aveva conosciuto a Londra nel 1930, quando entrambi frequentavano la sala di lettura del British (GERBI, S., 2011, p. 242-243). Che il libro tratti anche di autori inconsueti, di poeti e pittori, di altorilievi di fonti battesimali e di portali di chiese e perfino di oggetti d'arredo, induce a pensare anche alle ricerche animate da Aby Warburg; ma è somiglianza priva di riscontri concreti, dato che quando nel '30 e nel '32 visitò Amburgo (GERBI, A., 1993, p. 132-140, 276-284), Gerbi non ebbe contatti con la Kulturwissenschaftliche Bibliothek; né poté averne cognizione a Londra, dove l'Istituto fondato da Aby Warburg si trasferì solo nel '34, quando Gerbi n'era già ripartito.

La più viva associazione che si presenta alla mente di chi oggi legga l'Adamo ed Eva è con la storiografia delle idee', con la quale però nel '33 Gerbi non aveva ancora avuto contatti. Arthur Lovejoy pubblicherà $\mathrm{La}$ Grande Catena dell'Essere nel '36, mentre le sue proposte storiografiche avranno risonanza internazionale dal '40, con l'uscita del Journal of The History of Ideas. Ma poiché le affinità tra l'Adamo ed Eva e la storia delle idee non sono di superfice, interrogarsi su di esse non è avventuroso; anzi, il fatto che al tempo dell'Adamo ed Eva Gerbi ancora non conoscesse la storia delle idee dimostra che quando, negli studi di americanistica iniziati negli anni Quaranta, ne terrà conto, non farà se non ulteriormente coltivare interessi che aveva già autonomamente sviluppato nell'ambito dell'impianto storiografico crociano.

L'Adamo ed Eva esamina in opere d'un gran numero di filosofi, teologi, poeti, artisti ecc. le concezioni della Caduta carnale, studiate come variazioni d'un unico concetto base, $\mathrm{l}^{\prime}$ "[...] ipotesi beverlandiana” e in una ricerca modulare «a organetto» (GERBI, A., 1983, p. 4), sempre aperta a nuove analisi di altri filosofi, teologi ecc. È un'impostazione che ricorda quella di Lovejoy, che propone d'esaminare, in una cornice di storiografia soprattutto filosofica, un'unica idea in un gran numero di opere di filosofi, artisti, religiosi, scienziati, uomini politici ecc.: «[...] credo che sia innanzitutto necessario riconoscere che è nella storia della filosofia che si trova il terreno comune, ovvero il punto di partenza del maggior numero e delle più fondamentali e penetranti idee; specialmente dei fondamenti concettuali che si manifestano in altre regioni della storia intellettuale» (LOVEJOY, 1982, p. 35-36). In quell'amplissimo ambito, lo storico delle idee isola un'“[...] idea-unità

${ }^{9}$ Vari studiosi, in particolare RONCHETTI, 2002, 2007, 2009 e MELIS, 2000, si sono occupati dei rapporti di Gerbi con la storia delle idee, ma per gli studi di americanistica. 
elementare", «[...] per studiarla separatamente» (ivi, p. 38) dalle altre e delinearne diffusione e mutamenti morfologici «attraverso più d'una, anzi, in ultima analisi, attraverso tutte le sfere della storia in cui essa figura in misura notevole, sia quella della filosofia, oppure della scienza, della letteratura, dell'arte, della religione o della politica» (LOVEJOY, 1966, p. 21); e ciò in una ricerca che si spinge in «[...] campi che sembrano lontanissimi [dall'] argomento iniziale» (LOVEJOY, 1982, p. 34) e che, come "l'organetto" di Gerbi, è sempre espandibile.

Come si vede, oggetto ("ipotesi beverlandiana" e "idea-unità elementare") e metodo (gran varietà di fonti e impianto modulare) appaiono notevolmente somiglianti. E che si tratti d'affinità significative lo dichiara Lovejoy stesso, che nel 1946, ringraziando Gerbi d'avergli inviato Viejas Polémicas sobre el Nuevo Mundo (GERBI, A., 1946), gli scriverà:

It [il volume Viejas Polémicas] is of peculiar interest to me personally [...] because of its method - the connected study of the history of a single idea, or thesis, through all its principal phases and ramifications and interactions with other ideas, including those antitehetic to it [...] There is a special pleasure in finding a scholar in another country working so fruitfully in a field in which one has oneself labored. (ASI-BCI-P-GERBI, faldone 53).

E poiché il metodo dell'Adamo ed Eva è sostanzialmente il medesimo che nei successivi lavori d'americanistica, appare legittimo "anticipare" al '33 il giudizio col quale nel '46 Lovejoy, per così dire, associa Gerbi all' "History of Ideas Club» di Baltimora (nel 1960, quando a Cambridge si riunirà per la prima volta l'«International Society for the History of Ideas», Gerbi sarà, insieme a Paolo Rossi, uno dei due soli italiani presenti; Rossi, 1982, p. 14).

La legittimità d'“anticipare" all'Adamo ed Eva il giudizio di Lovejoy trova conferma negli «incrementi testuali» che il figlio Sandro ha tratto dal materiale che, dopo il '33, il padre era venuto raccogliendo, e che ha reso disponibili nell'edizione da lui curata ${ }^{10}$. Molti di quegli incrementi testuali sono riferiti a Lovejoy e ad altri studiosi variamente riconducibili alla storia delle idee, come Gilbert Chinard, George Boas, Bentley Glass, Owsei Temkin, William L. Straus Jr., Alexander Gode-von Aesch, Ernest Lee Tuveson.

\section{Qui esamino solo gli incrementi testuali riferiti a Lovejoy.}

\footnotetext{
${ }^{10}$ Un'avvertenza premessa da Sandro Gerbi alla seconda edizione dell'Adamo ed Eva informa che "le "stampelle" ( $(\mathbb{D})$ racchiudono gli incrementi testuali inseriti dall'Autore successivamente all'uscita della prima edizione (1933)» (GERBI, A., 2011, 12; grassetto mio). Per notizie sulla genesi e la storia del libro e sui criteri delle nuova edizione vd. GERBI, S., 2011.
} 
a) Nell'Adamo ed Eva Gerbi aveva osservato che le scoperte microbiologiche sei-settecenteche di Reinier de Graaf e Antoni van Leeuwenhoek avevano fornito un argomento alla teoria agostiniana dell'ereditarietà del Peccato: poiché nelle gonadi di Adamo erano presenti come microscopici homunculi (gli spermatozoi) i suoi tre figli, allora, per l'infinita divisibilità della materia, nelle ancor più microscopiche gonadi di Caino, Abele e Set homunculi «[...] dovevan esser[ci, sotto forma di] altri homunculi più piccini ancora», i nipoti di Adamo; così, di generazione in generazione, «[...] tutti eravamo realmente, personalmente, nei lombi, ora detti più propriamente testicoli, del primo padre Adamo» (p. 215), e quindi tutti peccammo con lui. Inoltre, esponendo la concezione di Fludd, che «[...] ogni cosa prima del peccato fosse creata e qua talis perfetta» e che Adamo avesse perso lo stato perfetto e precipitato "[...] "sé stesso nel circolo della riproduzione"» solo quando pretese di «[...] “creare ambiziosamente altri uomini, come Dio”» (p. 130), Gerbi aveva osservato come tale concezione, che svuota la Caduta di contenuti morali e la riduce a neutro abbandono della perfezione naturale, venga ripresa dalla visione secentesca della bontà naturale, per la quale tutte le leggi di natura sono buone; dunque "[...] bene ha fatto Adamo a fare quello che ha fatto", poiché con l' «Evae cognitio» si limitò a seguire «[...] la suprema legge di Natura, il più dolce e il più forte dei suoi comandi» (p. 131).

Secondo l'incremento testuale posto da Gerbi nella nota nr. 4 alle pagine 215-216, entrambe le considerazioni trovano riscontro nei «[...] corollari filosofici delle scoperte dei microscopisti» (p. 216n) messi in luce ne La Grande Catena dell'Essere. Lovejoy osserva infatti che la scoperta dei microrganismi alimentò sia l'immagine pessimistica del «[...] desolante spettacolo di un universale parassitismo, di una vita che dovunque si pasce della vita», immagine consona all'argomento degli homunculi sulla propagazione ereditaria del Peccato e dell'umanità massa damnata, sia quella ottimistica della "[...] prodigiosa fecondità della natura», consona invece alla concezione della bontà delle leggi naturali (LOVEJOY, 1966, p. 257, 258).

ß) Nell'Adamo ed Eva Gerbi aveva citato un'affermazione di Bergson, «Le souvenir du fruit défendu est ce qu'il y a de plus ancien dans la mémoire de chacun de nous, comme dans celle de l'humanité» (BERGSON, 1970, p. 981), e osservato che questo "sentimento di una colpa originaria" - che non è solo di ebrei e cristiani, ma anche di altre credenze, di tradizioni non confessionali e soprattutto della vasta "[...] folla confusa degli umili e dei pii» - "universalizza" e insieme "depotenzia" la Colpa, inducendo a considerare Adamo ed Eva come simboli di un'umanità fragile ed esposta a un' «eterna Caduta», ma non 
inesoratamente damnata (p. 200, 201). È a queste considerazioni che Gerbi si riferisce con l'incremento testuale della nota nr. 1 a p. 201, che rinvia a un saggio del 1940, Coleridge and Kant's Two Worlds (LOVEJOY, 1948, p. 254-276), nel quale Lovejoy mostra la presenza nel poeta inglese di un analogo rapporto tra "universalizzazione e "depotenziamento". Secondo Coleridge, spiega Lovejoy, anche se "the Article of Original Sin"» è "the central truth» del Cristianesimo, tuttavia «it is "no tenet first introduced and imposed by Christianity"», ma esprime un carattere "universale" della moralità umana, "an Axiom of Faith in all classes [...] acknowledged in all ages" "; "universalizzazione” che non implica necessariamente l'agostiniana "hereditary transmission to all the descendants of Adam of the taint arising from the sin of our first parents», bensì "depotenzia" la colpevolezza dell'umanità: "[...] since we are not Adam, it is not his sin [...] for which our wills are responsible» (ivi, p. 268, 269).

$\gamma)$ Nell'Adamo ed Eva Gerbi aveva esaminato gli aspetti contrastanti del pensiero e della personalità di Milton: puritano, ma sensibilissimo al fascino muliebre; inorridito della disobbedienza a Dio, ma spirito ribelle che simpatizza con Satana e considera l'amplesso peccaminoso di Adamo ed Eva anche come testimonianza della libertà e dignità umane: se Adamo non avesse avuto libertà e ragione, scrive nell'Areopagitica, non avrebbe peccato, ma sarebbe stato «[...] "un Adamo artificiale, un Adamo da teatro di marionette!"” (p. 147). Queste concezioni contrastanti, aveva osservato Gerbi, erano presenti già nella Gnosi; ma in Milton sono vivissime, e lo portano «[...] ad attenuare, direi quasi a riscattare la colpa di Adamo» (p. 59), animando "[...] le parti più belle del Paradiso Perduto» (p. 147). Ben lo mostra la descrizione di Adamo che mangia il pomo e cede al fascino di Eva non perché ingannato da lei o dimentico del divieto divino (p. 148), ma perché "sceglie" di farlo:

\section{[...] he scrupled not to eat}

Against his better knowledge, not deceived,

But fondly overcome with female charm. (lib. IX, vv. 998-1000).

Che questa scelta conferisca dignità al Peccato lo s'avverte nella descrizione del primo amplesso, da cui traspare che l'intimo telos dell'erotismo è l'amore vero e legittimo, che attrae e comprende in sé la carnalità; dopo la lussuria iniziale,

[...] in lust they burn. (lib. IX, v. 1016), 
l'erotismo si muta infatti in lenimento della colpa:

[...] they their fill of love and love's disport

Took largely, of their mutual guilt the seal,

The solace of their sin... (lib. IX, vv. 1042-1044; corsivo di Gerbi);

lenimento ch'è premessa d'amore coniugale legittimo (p. 148):

Hail wedded love! mysterious law, true source

Of human offspring, sole propriety

In Paradise of all thing common else. (lib. IV, vv. 750-753).

Gerbi cita (p. 61) anche gli ultimi versi del poema, che descrivono non una cacciata, ma una serena uscita dall'Eden, con i due peccatori, ormai consapevoli della futura Redenzione, che si avviano a prender possesso del mondo e dare inizio alla storia:

Some natural tears they dropped, but wiped them soon;

The world was all before them, where to choose

Their place of rest, and Providence their guide.

They, hand in hand, with wand'ring steps and slow

Through Eden took their solitary way. (lib. XII, vv. 645-649; corsivo di Gerbi)

A questi temi miltoniani fa riferimento l'incremento della nota nr. 1 di p. 59, che rinvia a un saggio del 1937, Milton and The Paradox of The Fortunate Fall (LOVEJOY, 1948, p. 277-295), dove Lovejoy commenta l'espressione "felix culpa", usata dalla patristica per significare che dalla Caduta sarebbe derivato il maggior bene della Redenzione. Nel saggio Lovejoy cita (ivi, p. 277) i versi nei quali Adamo, dopo che l'arcangelo Michele (lib. XII, vv. 462-465) gli ha profetizzato la storia sacra dell'umanità, enuncia quello che Lovejoy chiama il "paradosso" (e Gerbi gli "aspetti contrastanti") della Caduta: la Disobbedienza fu insieme un male e un bene:

\author{
[...] full of doubt I stand, \\ Whether I should repent me now of sin \\ By me done and occasioned, or rejoice \\ Much more, that much more good thereof shall spring. \\ To God more glory, more good will to men \\ From God, and over wrath grace shall abound. (lib. XII, vv. 473-478).
}

$\delta$, $\varepsilon)$ Nell'Adamo ed Eva Gerbi aveva scritto che Ambrogio non ha una "chiara teoria del Peccato», del quale dà tre diverse spiegazioni: in una, d'ordine 
spirituale, la causa è la «superbia animi», della quale la «delectatio corporalis» è l'effetto; nelle altre due, d'ordine invece sensibile, la causa sono o la concupiscenza, o l'emozione amorosa provata da Adamo quando Eva lo ammansì «[...] "con il cibo e con la forza del proprio amore" (p. 40-41). Gerbi aveva osservato che quest'ultima spiegazione apre la strada a un'idea «bonaria» (p. 41) della Caduta, e nell'incremento testuale della nota nr. 1 a p. 41 fa riferimento, oltre che di nuovo a Milton and the Paradox of the Fortunate Fall, a un saggio del 1942, The Communism of St. Ambrose (Lovejoy, 1948, p. 296-307), nel quale anche Lovejoy osserva che Ambrogio dà tre diverse spiegazioni della Caduta: in una, influenzato dalla concezione pagana dell'Età dell'oro, afferma che nell'Eden non esisteva la proprietà e che causa della Caduta fu la "prima avaritia», dalla quale scaturirono cupidigia e lussuria; secondo le altre due, Adamo disobbedì o per «superbia or insolentia», oppure per «appetentia voluptatis» (ivi, p. 296, 296n). Come si vede, le interpretazioni di Lovejoy e Gerbi sono sensibilmente affini: entrambi rilevano la presenza di tre diverse spiegazioni della Caduta, e due di esse (superbia animi e concupiscentialappetentia voluptatis) sono identiche.

\section{L'Adamo ed Eva e Croce}

Gli studi di americanistica condotti da Gerbi a far tempo dal soggiorno in Perù (dove, per sottrarsi alle leggi razziali, si trasferì nel 1938 e rimase sino al 1948) furono certo influenzati dai contatti con la storia delle idee, ma la prospettiva storiografica restò saldamente ancorata al magistero crociano. Poiché non è qui possibile esaminare l'insieme della sua produzione, come sopra mi sono ristretto all'esame delle affinità tra l'Adamo ed Eva e alcuni scritti di Lovejoy, così ora mi restringo ad esaminare le testimonianze esplicite dell'influenza di Croce sull' $A$ damo ed Eva. Aggiungo solo qualche cenno a scritti di Gerbi successivi al 1946 - data del contatto diretto con Lovejoy -, per mostrare che rimase convintamene fedele ad una scelta di Croce "storico" fatta appena ventenne, quando recensendo i primi due volumi di Conversazioni critiche aveva scritto che «[...] "spicca in questi articoli quella che $[\ldots]$ mi pare la qualità fondamentale di Croce: più che meramente filosofico, più che meramente critico, il suo spirito è storico" ${ }^{11}$.

Un documento esemplare del fatto che Gerbi restasse saldamente crociano è la lettera che il 16 ottobre 1957 scrive ad Antonine Tibesar, segretario della "Conference on the History of Religion in the New World", nella quale, per declinare l'invito a partecipare a un'opera storica collettiva, si

${ }^{11}$ Traggo la citazione da TREVES, 1983, p. xxxix; i punti sospensivi sono di Piero Treves. 
richiama alla metodologia che, dichiara, «j’ai hérité de mon maître Benedetto Croce» (ASI-BCI, P-GERBI, Faldone 56), e con motivazione schiettamente crociana scrive che la ricerca storica nasce da un problema che lo studioso si pone individualmente: «Il est même contradictoire à la conception de l'histoire comme "réponse" à une question née dans l'esprit d'un historien, - et pourtant unique, individuelle, irrépétible, - que l'on puisse se mettre à plusieurs pour la formuler». Di questo principio, sei anni prima Gerbi aveva fatto un'esposizione, ch'era anche intima adesione, in un testo apparso anonimo, ma molto significativo già per il luogo in cui venne stampato: l' "Appendice» all'antologia di suoi scritti che Croce pubblicò nel ' 51 da Ricciardi, della quale Gerbi fu co-curatore ${ }^{12}$. Poiché il volume ricciardiano - quasi un testamento spirituale, preparato da Croce quando già era stato colpito da un ictus, a ridosso della guerra e nell' "[...] atroce tristezza del tramonto contornato da stragi e distruzioni di tutto quanto tenevamo caro e sacro al mondo» - mirava a dare "[...] una immagine, compendiosa bensì ma intera, dell'opera sua [di Croce]» (CROCE, 1951, p. 3, c. 3r), ci si attenderebbe che l' "Appendice» dedicasse alla filosofia un'attenzione proporzionale al peso (circa un terzo) ch'essa ha nel volume. Invece Gerbi sorvola sulla parte filosofica per insistere soprattutto sul lavoro "storico" fatto da Croce in ciascuna delle quattro forme. In estetica, scrive, Croce ha attuato la «[...] riforma della storia della poesia e dell'arte» (ivi, p. 1178; corsivo mio). In filosofia, le monografie Ciò che è vivo e ciò che è morto della filosofia di Hegel e La filosofia di Giambattista Vico mostrano che Croce non si è fermato agli «enunciati generali», ma è sceso "nel determinato e nel concreto», mettendo in luce il «rapporto storico» che le sue «conclusioni» hanno «con la storia delle questioni» da cui si originano, sí da porre i lettori in condizione d'inserirle "[...] in rinnovati rapporti storici» (ivi, p. 1177,1179; corsivi miei). In ambito pratico, «[...] le teorie politiche e morali della Filosofia della pratica hanno la loro corrispondenza» (ivi, p. 1180-1181) nelle grandi opere storiche su La rivoluzione napoletana del 1799, la Storia del Regno di Napoli, la Storia dell'età barocca, la Storia d'Italia e la Storia d'Europa. Queste valutazioni di Gerbi sulla preminenza di Croce "storico" su Croce "filosofo" sono prossime a quelle poi ampiamente argomentate da Eugenio Garin, che mostrerà come la radice della longevità culturale di Croce e del perdurante interesse ch'egli suscita siano «[...] non [...] le sue quattro forme», ma l'«[...] aver imposto definitive interpretazioni di avvenimenti e di periodi storici» (GARIN, 1962, p. 224).

${ }^{12}$ CROCE (1951); nel frontespizio il volume appare a cura di Croce, che però nell' "Avvertenza» ringrazia Gerbi per aver con lui «curato il testo» (c.3r). 
Oltre che in punti specifici, come il suggerimento (di Croce e di Alessandro Casati) che Gerbi prendesse in esame anche il "[...] "beverlandismo" dei Càtari» (p. 74n), l'influenza di Croce "storico" pervade tutto l'Adamo ed Eva; ma qui, dicevo, esamino solo i punti nei quali si manifesta in modo esplicito.

Per illustrare come nel Rinascimento la Caduta carnale venisse interpretata in Europa settentrionale, Gerbi, s'è veduto, si sofferma anche sulle arti figurative. Osserva che «[...] intorno al 1520 [...] si comincia nei paesi tedeschi a dotare generosamente Adamo ed Eva dei loro attributi sessuali», e collega questa tendenza al fatto che «[...] proprio in quegli anni Lutero, e poi Calvino, rinnovando ed inasprendo la tesi agostiniana, facevan del peccato originale un peccato di concupiscenza [...] e aprivano [...] il varco ad ogni sorta di imprecise e impure meditazioni» (p. 113). A questo proposito Gerbi cita l'edizione dell'Elogio della follia che Croce curò nel 1914 (e che riproduce le figure dell' «[...] esemplare dell'edizione frobeniana del 1514, i cui margini il giovane Holbein, in una decina di giorni dell'anno 1515, ornò di disegni a penna» (CROCE, 1914, p. VI), e si sofferma sul disegno che raffigura Adamo con gli attributi sessuali ben visibili; disegno col quale, scrive, Holbein «[...] illustra questo passo dell'Elogio della follia d'Erasmo: "il grande Architetto dell'Universo proibì a quella bellissima coppia di sposi, che primi egli aveva formati e uniti in matrimonio ... di gustare del frutto dell'albero della scienza del bene e del male"»(ERASMO, 1914, p. 144, figura 2). Il riferimento ad Erasmo è invero improprio ${ }^{13}$, ma ciò che qui importa non è che Gerbi sia impreciso su Erasmo e la Riforma, bensì che prenda Croce a modello per integrare ricerche storiche e ricerche iconografiche; integrazione frequente

\footnotetext{
${ }^{13}$ L'omissione introdotta da Gerbi con punti sospensivi induce a fraintendere il passo di Erasmo, che integro suona così: «Questo legislatore [«Gesù Cristo, il quale è la sapienza del Padre»] vieta loro [agli uomini] di prepararsi allorché dovranno comparire innanzi ai tribunali de’ re e de’ presidi; non vuole che pensino al dimani, né che osservino la misura del tempo, per timore che, confidando nella loro sapienza, non s'abbandonino interamente alla sua provvidenza. Fu pure per questa ragione, che il grande Architetto dell'universo proibì a quella bellissima coppia di sposi, che primi egli aveva formati e uniti in matrimonio, proibì, dico, di gustare del frutto dell'albero della scienza del bene e del male, sotto pena della sua disgrazia e della sua morte. Gran prova che la scienza è il veleno della felicità» (ERASMO, 1914, p. 143-144). Il chiaro senso è che per Erasmo la Caduta non fu carnale: uniti in matrimonio dall'Eterno, Adamo ed Eva potevano ben avere rapporti sessuali, ma non voler divenire scientes bonum et malum; l'Elogio della follia andava casomai citato come occorrenza "anti-beverlandiana". In questo contesto, alcune considerazioni di Gerbi sono imprecise anche dal punto di vista storico: nella «Prefazione» Croce aveva chiaramente scritto che Holbein illustrò l'esemplare dell' Elogio non «intorno al 1520", ma "in una decina di giorni dell'anno 1515», due anni prima dell'affissione di Wittenberg; nessuna relazione specifica, dunque, tra la "generosa raffigurazione degli attributi sessuali" di Adamo e la predicazione di Lutero; tanto meno con quella, ancor più tarda, di Calvino.
} 
in Croce, come mostra, per ricordare un solo esempio, la ricerca su I ritratti di Giulia Gonzaga.

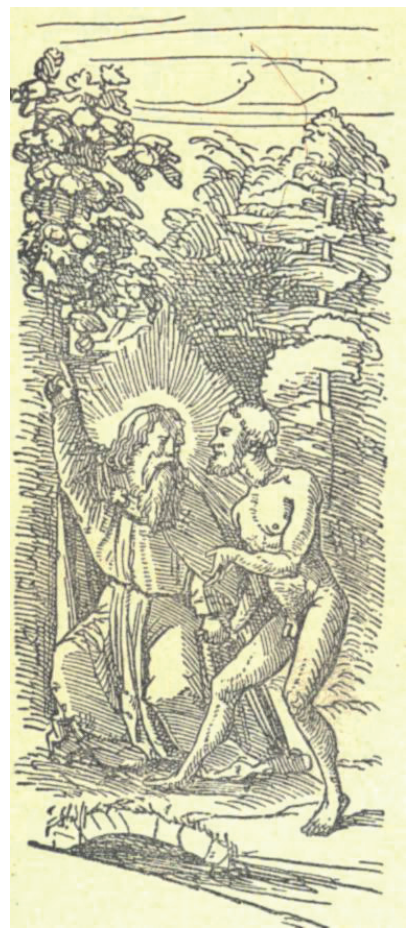

Quasi per intero riferibile a Croce "critico" è la trattazione che Gerbi fa del "beverlandismo" di Baudelaire, collegandolo alle polemiche del poeta "contro il Progresso» (p. 188), «[...] contro l'Amore [romantico] e [contro] la Natura pretesa buona» (p. 192). Baudelaire, scrive, considera Satana un «amico degli uomini» (p. 193) e vede nel Peccato originale sia la "prova storica che la natura [non è buona, ma] corrotta» (p. 192), sia l'«[...] immagine riassuntiva dei mali e dei vizi infiniti» (p. 188) di un'umanità incapace di ogni progresso; egli rifiuta la concezione romantica dell'amore e sostiene che «l'amore è male» e che il nostro stesso «[...] "modo di generare [è] un segno del peccato originale" ", come provano il fatto che "[...] "non possiamo fare l'amore che con degli organi escrementizi"» e che "la voluttà unica e suprema dell'amore consiste nella certezza di fare il male” (p. 191, 192). 
Sono giudizi e osservazioni che ripercorrono il saggio Baudelaire pubblicato da Croce ne «La Critica» nel 1919 e ristampato nel 1923 in Poesia e non poesia (CROCE, 1923), e nel quale tutti gli elementi poi ripresi da Gerbi sono presenti: che Baudelaire esalti «Satana-uomo» (ivi, p. 262), pietoso dei miseri e degli umili; che riprenda "la dottrina del "peccato originale" " per polemizzare col "[...] cosiddetto "progresso", come era stato ideato nel secolo decimottavo e rivestito di romantici colori dalla ideologia liberale del decimonono» (ivi, p. 252); che sbeffeggi la romantica "religione dell'amore», protestando che «[...] "la voluttà unica e suprema dell'amore è riposta nella certezza di fare il male” (ivi, p. 254); che al «[...] culto della natura: tanto della "natura" come l'aveva intesa il secolo decimottavo, quanto di quella dei romantici», ribatta che «[...] la natura intera [...] partecip[a] del "peccato originale"» (ivi, p. 255). È inoltre da presumersi che Gerbi conoscesse la nota su La gioia del male (1916), dove Croce tratta del «[...] piacere del frutto proibito» (CROCE, 1951, p. 651) e cita anche Baudelaire ${ }^{14}$.

L'Adamo ed Eva contiene un esplicito riferimento a Croce "filosofo" delle quattro forme e della dialettica degli opposti. Gerbi situa infatti l'istinto sessuale all'infimo dell'"economica", passibile di divenire preda, ad es. nella dottrina cattolica, di una dialettica tra gli "opposti" di vita e morte: «L'atto sessuale è peccato ed è morte quando il singolo sia di fronte a Dio; l'atto sessuale è vita, elementare ma irrefutabile, $[. .$.$] quando l'uomo sia tra i suoi simili, nelle società$ del genere umano. L'istinto che lo determina, considerato con rigore filosofico, è la prima forma dell'attività "meramente economica" (Croce), la forma più elementare, la più vicina alla mera animalità (come la Politica è la più complessa e più vicina alla pura eticità), e perciò sempre in pericolo d'essere espulsa dai rigoristi, di vedersi negata ogni spiritualità» (p. 31; corsivi miei). Posizione sulla quale recensendo l'Adamo ed Eva Croce pone il sigillo della sua filosofia: prima ancora di lodare il libro per la «molta erudizione» storica e il «brio di svariate osservazioni» e di avanzare la riserva che Gerbi avesse «forse esagerato» l'importanza dell'“ipotesi di Beverland” (CROCE, 1933, p. 377, 378), Croce approva l'impostazione filosofica della relazione tra sessualità e moralità:

[...] egli [Gerbi] giustamente vede [...] nel concetto della peccaminosità dell'atto sessuale e nel superamento di questo concetto, il riflesso del più

\footnotetext{
${ }^{14}$ Un altro rimando a Croce "critico" si ha dove, trattando della teoria di Novalis, che assimiglia l'attività spirituale al mangiare ("Godere con lo spirito è simile a nutrirsi col corpo"»; 167n), Gerbi rinvia alla recensione scritta da Croce sul critico letterario Jean Pommier, che aveva interpretato il romanzo $A$ la recherche du temps perdu come manifestazione artistica della pulsione di Marcel Proust a "mangiarsi" come madelaines le emozioni e i rapporti familiari e sociali. La concezione dell'arte come surrogato del manicare viene liquidata da Croce come «"désarroi"» (CROCE, 1939, 450).
} 
generale problema di vita e moralità. Questo problema rimane insolubile sempre che si presenti, secondo che si è stati soliti di formularlo, e ancora lo si formula, come problema dei rapporti tra spirito e natura; ma (e questo il Gerbi ammette da sua parte) si avvia a soluzione quando si converte nell'altro dei rapporti tra due forme dello spirito stesso, quella meramente volitiva o economica e quella etica, che, come tali, non sono parallele o disparate, ma opponentisi e trapassanti l'una nell'altra, e perciò formano unità organica concreta. (ivi, p. 377).

La relazione tra istinto sessuale e moralità è ripresa in un altro passo dell'Adamo ed Eva, che cito per esteso e al quale Gerbi apporrà una nota riferita a Croce "storico" e "oppositore politico". Il passo, che commenta la cacciata dall'Eden, usa una metafora per distinguere l'attività sessuale matura e consapevole, che "germoglia a fior dell'onda", da quella che permane "nel buio dell'abisso": con la cacciata dall'Eden, scrive Gerbi,

Dio resta senza gli uomini, e gli uomini restan senza Dio. Per questo, forse, il Diavolo non ci ha più lasciati. L'angoscia del male non ci ha più lasciati. Del mito e del dogma in senso proprio avanzano ormai solo dei residui verbali. Ma la sua essenza è scesa sino in fondo alla coscienza di noi tutti, vi si è posata come un relitto di naufragio si posa sul fondo del mare, è diventata la nostra stessa coscienza, che da quel buio d'abisso sale, ramifica e germoglia a fior dell'onda². (p. 202).

Ed ecco il testo della nota nr. 2, che chiude il passo (il quale, ricordo, è del 1933) e viene aggiunta da Gerbi nel 1937, quando legge ne "La Critica» l'anticipazione della prima parte de La storia come pensiero e come azione (il celebre volume omonimo uscirà nel 1938): «Cf. B. Croce, La storia come pensiero e come azione, I, in «La Critica». v. 35, 1937, p. 9 》" (miei i grassetti).

L'occasione immediata del rinvio di Gerbi è che quella pagina de «La Critica" contiene una metafora simile alla sua, nella quale Croce relega "negli abissi" la pseudo-storiografia fascista e nazista. La trascrivo qui, ma avvisando che la piena comprensione della metafora di Croce (e dell'incremento testuale di Gerbi) richiederà un passo indietro, per collocarla nel contesto della rimeditazione del proprio pensiero pratico avviata da Croce dopo il colpo di Stato fascista. Nella metafora, Croce condanna la pseudo-storiografia del fascismo e del nazismo perché verte non su problemi politici, morali, culturali (quindi su oggetti "immateriali"; crocianamente: spirituali), ma su feticci di cose: sostanzializzano la nazione e la razza, 
[...] attribuendo a loro quella realtà e quel valore che è solo delle azioni che lo spirito compie, delle opere sue, politiche e morali, scientifiche ed artistiche, delle quali e non delle cose, che sono astrazioni e perciò non si svolgono, si chiede e s'indaga la storia. Sostanzializzate, e perciò materializzato in esse lo spirito, tarpategli le ali, prendono di necessità sembiante equivoco e si prestano a far da recipienti a quanto di morboso o di mostruoso si divincola, qual serpe, nei bassi fondi dell'anima umana: libidine e istinto predace e violenza e ferocia e crudeltà, e poi disgusto del vivere, e acedia e brame di dissolvimento; di quanto l'uomo ricaccia in giù e preme col piede, innalzandosi ad attività spirituale, e che qui vien messo in libertà, e lasciato espandere, e morbosamente rimirato e vezzeggiato. (CROCE, 1937, p. 8-9).

Fatti uscir di catena dal fascismo e dal nazismo, quei mostri generano le «morbose e mostruose storie di cose mostruose e morbose [che] si manifestano ai giorni nostri come storie "nazionalistiche" o "razzistiche" ", delle quali molti esempi vediamo «[...] ai nostri giorni in Germania» (ivi, p. 9).

Qui, dicevo, occorre una breve digressione sull'evoluzione del pensiero politico di Croce, che, com'è noto, prima della dittatura fascista aveva teorizzato la netta separatezza tra morale e politica. Nel terzo volume della filosofia dello spirito, Filosofia della pratica. Economica ed Etica (1909), ad es., aveva scritto che la politica non obbedisce a criteri morali e non persegue se non l'utile, "[...] ciò che è corrispettivo soltanto alle condizioni di fatto, in cui l'uomo si trova» (CROCE, 1909, p. 219). Di questa separatezza era imbevuta la pugnace campagna condotta da Croce contro le interpretazioni razionalistiche, contrattualistiche e utilitaristiche dello Stato, per ribadire che «[...] la politica, come l'economia, ha leggi sue proprie, indipendenti dalla morale» (CROCE, 1916a, p. 241), e per polemizzare contro la «[...] mentalità del secolo decimottavo", che persiste "[...] in molta parte della società del secolo ventesimo» e lo induce a "[...] ripugnare alla teoria dell'autonomia della politica (come dell'autonomia dell'arte)»(CROCE, 1916b, p. 243). Di qui gli insistenti richiami a prendere atto che lo Stato è "forza" e "potenza", le irrisioni al pacifismo e all'umanitarismo, la legittimazione della guerra. Nel pieno della carneficina di Verdun, ad es., Croce, che pure era stato antinterventista, scrive che «[...] questa religiosa ecatombe, alla quale la vecchia Europa si è offerta fidente nell'avvenire e guardando ai figli dei figli», è l'occasione "per liberarci dai preconcetti astrattamente umanitarii» (CROCE, 1916a, p. 241) e dall'utopia socialista, che «[...] un tempo aveva sognato di stringere in alleanza i proletari di tutto il mondo» (CROCE, 1916b, p. 243). 
Ma nella rimeditazione sollecitata dalle "nuove esperienze di vita» (CROCE, 1938, p. vii) impostegli dalla dittatura fascista, Croce sviluppa una nuova concezione "etico-politica"” dell'agire umano nella storia, per la quale «[...] la moralità non crea la politica o l'utilità, come non crea le altre forme dell'attività umana, ma le comprende tutte e tutte le converte, in quanto adempiono al loro fine speciale, in azione etica" (CROCE, 1937, p. 32). L'azione politica non è più circoscritta all'utile, ma immessa nel «[...] complicato e delicato [...] processo dialettico onde il pensiero storico nasce da un travaglio di passione pratica, lo trascende liberandosene nel puro giudizio del vero, e, mercé di questo giudizio, quella passione si converte in risolutezza di azione» (CROCE, 1938, p. viii). E poiché in Croce giudizio storico e giudizio di verità coincidono, azione politica verace sarà solo quella che si compie nella "[...] "storia come storia della libertà"»(CROCE, 1937, cap. XII). Cade anche la legittimazione della guerra: Croce giudica quella nazifascista una "guerra orrenda» (CROCE, 1993, v. 2, p. 217), e in una lapide in memoria di una strage di civili scrive che le armate hitleriane sono composte da "[...] gente [...]/ nella quale orrenda si è discoperta / armata di tecnica moderna / la belva primeva» (ivi, v. 2, p. 274).

La parte de La storia come pensiero e come azione anticipata nel 1937 su «La Critica» è uno dei testi, nei quali il mutamento d'orizzonte riguardo ai rapporti tra politica e morale è espresso in modo straordinariamente incisivo. Croce ribadisce che ogni genuina opera storica è "storia contemporanea" , frutto «[...] del bisogno e della situazione presente» (CROCE, 1937, p. 4), ma che l'impulso pratico deve poi convertirsi nell'atto conoscitivo del "giudizio storico" (ivi, p. 7), il solo capace di conferire unità alla ricerca storica; unità che non è mai «in una cosa» (ivi, p. 7; corsivo mio), nella materialità dell'oggetto della ricerca, ma sempre e solo «[...] nel problema che il giudizio storico formula» (ivi, p. 7; corsivo mio). Scritti che si pretendano storici ma cerchino l'unità in "cose" (storia di un popolo, di una città, di un mare ...) non sono propriamente opere di storia, ma cronache o costruzioni che mescolano elementi storici e di fantasia, pensieri morali ed auspici politici. Ma se lavori di questo tipo possono anche risultare nobili, utili e piacevoli, e comunque non dannosi, «[...] il male comincia» quando con argomentazioni «senza capo né coda» (ivi, p. 8) ci si intestardisca, ad es., a voler comporre "storie nazionali", come quelle volte a rintracciare un' "unità della storia d'Italia”, materialmente intesa» (ivi, p. 8) in epoche, nelle quali il problema politico e morale dell'unità nazionale ancora non era sentito. Ma se la pretesa di fare tali "storie nazionali" «[...] è il male, non è il peggio, perché il peggio qui nasce dal sostanzializzare 
le cose» (ivi, p. 8), come nella storiografia nazionalistica del regime fascista e in quella razzistica del III Reich. Ed è qui che Croce usa la potente metafora, che condanna il nazifascismo come "mostruosa belva primeva".

Gerbi segue le orme di quest'evoluzione di Croce. Nel primo lavoro, $L a$ politica del settecento: Storia di un'idea $(1928)^{15}$, è ancora fermo alla posizione crociana dello Stato come potenza e critica Voltaire come tipico rappresentante della "mentalità del secolo decimottavo", che non separa la politica dalla morale e dunque non può capire «la passione creatrice dell'uomo di stato» (GERBI, A., 1928, p. 60); per Voltaire, «[...] la politica estera si riassume nel brigantaggio, nella frode, nella sopraffazione, l'interna nello spillare ai cittadini quanto più danaro si può», e la guerra è "[...] un mostro cruento, è male per definizione e chi la fa è per ciò solo un criminale» (ivi, p. 60, 61). Cinque anni dopo, nell'Adamo ed Eva, la separatezza della politica dalla morale è invece rifiutata, e la politica, s'è visto, viene considerata un'attività "più complessa che non il mero perseguimento dell'utile e più vicina alla pura eticită”.

Diversi e carichi di significati "storici", "politici" e "personali” gli aspetti della personalità di Gerbi che si compendiano nel pur brevissimo riferimento a Croce posto alla fine del passo «Dio resta senza gli uomini [...] a fior dell'onda ${ }^{2}$ ». Innanzitutto esso conferma che Gerbi guarda sempre e soprattutto a Croce "storico". La storia come pensiero e come azione è infatti insieme un trattato di metodologia storiografica e un manifesto etico-politico, denso di gravi riferimenti storici all'Europa, "[...] in molti paesi [della quale] gli ordini liberali, che furono il grande acquisto del secolo decimonono e sembrarono acquisto in perpetuo, sono crollati e in molti altri s'allarga il desiderio di questo crollo» (CROCE, 1937, p. 33). Gerbi mostra d'aver ravvisato nella metafora di Croce un'analogia profonda con la propria: come in quella di Croce le pseudo-storiografie fasciste e naziste sono parti di mostri abissali, frutti maligni e distruttivi, opposti alle autentiche opere storiche, che si compiono nella luce solare della consapevolezza conoscitiva e morale, così nella metafora di Gerbi l'istinto sessuale che permane negli abissi del profondo ${ }^{16}$ genera ramificazioni morbose di "peccato e morte", mentre se affiora al fior dell'onda della consapevolezza morale è "positività e vita”. In entrambe le metafore vi è poi il rifiuto d'insuperabile separatezza tra sfera morale e economico/istintuale: Croce respinge le reificazioni di nazione e

${ }^{15}$ GERBI, A., 1928. Il titolo non tragga in inganno: nel lavoro non ci sono ancora le novità che rendono l'Adamo ed Eva affine alla storia delle idee.

${ }^{16}$ L'espressione "profondo" non è qui impropria, poiché nella tavola riepilogativa appare il termine "psicanalisti» e nell' Adamo ed Eva Freud è citato tre volte. 
razza e che la storiografia sia strumento di politiche dittatoriali, Gerbi rifiuta le concezioni mortifere della sessualità e applica alla vita sessuale ciò che nel ' 51 dirà della crociana filosofia pratica: che mostri «[...] il legame tra le due forme di volontà, l'utilità o mera volontà di vita e la morale, di cui la seconda sorge non come abolizione e distruzione, ma come critica ed educazione ed innalzamento della prima» ([GERBI, A.], p.1178). In sintesi, questo era stato anche il nocciolo teorico dell'Adamo ed Eva, dove Gerbi aveva descritto lo svolgersi dell'insopita tensione tra il sesso come "vita" e come "morte" nella fenomenologia storica delle concezioni della Caduta, che nel bimillenario tessuto d'esperienze e dibattiti s'erano evolute non già sino all'utopia d'un inscalfibile principio del sesso come vita, ma almeno a dare via via spazio a vissuti più maturi che non, come s'è veduto aver sostenuto Filone, la riduzione dell'attività sessuale a mero "[...] "piacere fisico, che è radice prima di iniquità e di prevaricazione"» (p. 22).

Il riferimento al testo crociano del 1937 mostra anche che Gerbi nutrì il suo antifascismo «[...] "della prima ora” ${ }^{17}$ dei giudizi politici e morali espressi da Croce "storico" e, in quanto "storico", "oppositore" del nazifascismo; egli fu tra «i testimoni di quegli anni [che potevano] misurare nella loro memoria il conforto e l'efficacia recati» (CONTINI, 1972, p. 63) dalla resistenza spirituale esercitata da Croce in libri possenti, come la Storia d'Europa e $\mathrm{La}$ storia come pensiero e come azione.

Nel rinvio alla metafora crociana vi è anche un aspetto più intimo della personalità di Gerbi: leggendo nel 1937 la critica di Croce al razzismo hitleriano, non poteva non colorarla anche di personale profonda angoscia per le persecuzioni antisemite in Germania. E non solo perché, come Croce, la Germania l'aveva amata, ma anche per il gravissimo timore che la politica di Hitler per l'annessione dell'Austria gli suscitava: l'estensione delle leggi razziali di Norimberga (1935) agli ebrei austriaci, che avrebbe fatalmente colpito la famiglia della fidanzata Herma Schimmerling (si sarebbero sposati nel 1940). L'anno dopo, con l'Anschluß e le leggi razziali di Mussolini, il trionfo dei mostri abissali divenne realtà: per Gerbi sarà l'esilio in Perù, dove Raffaele Mattioli, presidente della Banca Commerciale Italiana nella quale lavorava, lo trasferì nel 1938 per sottrarlo alle persecuzioni razziali; per i genitori della donna amata sarà la morte: la madre, Ernestine Loewy, morì assassinata ad

${ }^{17}$ Gerbi fu "antifascista della prima ora” (GERBI, S., 2002, 104; vd. anche 58-61) per vicinanza a Croce, per ambiente familiare (era imparentato con il deputato socialista poi fuoriuscito Claudio Treves, padre di Paolo e Piero, con il filosofo del diritto Alessandro Levi, col pittore Carlo Levi e con Carlo e Nello Rosselli), per frequentazioni amicali (Eugenio Colorni, Leopoldo Gasparotto, Alessandro Casati, Guido Morpurgo-Tagliabue e altri) e di lavoro (Raffaele Mattioli, Ugo La Malfa e altri). 
Auschwitz; dalle poche e incerte testimonianze che abbiamo, sembra che il padre, Otto, sia caduto combattendo con i partigiani jugoslavi (GERBI, S., 2002, p. 75; GERBI, S., 2009, p. 45).

\section{Conclusione}

A riguardare l'Adamo ed Eva e quanto s'è detto di Lovejoy e Croce, si constata che le affinità con la storia delle idee non intaccano l'intrinseco legame di Gerbi con la metodologia storiografica crociana. Nella narrazione della lunga e mai conclusa fatica d'imparare a leggere nella Caduta la «[...] metafora della nostra fragilità, della nostra mortalità morale» (p. 202), Gerbi si pone nell'alveo della tradizione storiografica che, attraverso la revisione di Croce, si rifa alla concezione hegeliana della storia come svolgimento diacronico.

Per determinare la distanza metodologica che separa l'Adamo ed Eva dalla storia delle idee, in conclusione è opportuno svolgere, a titolo d'esempio, un sintetico esame comparativo tra l'Adamo ed Eva e il saggio Milton and The Paradox of The Fortunate Fall, nel quale, s'è veduto, l'affinità "spontanea" tra Gerbi e la storia delle idee è tangibile. Lovejoy, secondo il quale il "paradosso" "was no invention, or discovery, of Milton» (LOVEJOY, 1948, p. 279), dopo aver citato diciassette versi (vv. 462-468) del XII libro di Paradise Lost (tra i quali i sei, vv. 473-478, che ho sopra trascritto), nel $\$ 1$ passa ad elencare le occorrenze di «[...] earlier phrasings of the same idea» (ivi, p. 279); la prima occorrenza sono sedici versi - sette nell'originale francese e nove nella traduzione inglese di Joshua Sylvester (1563-1618) - del poema di Guillaume de Salluste Du Bartas (15441590) La Sepmaine; ou, Creation du monde, dove (ivi, p. 280) l'idea-paradosso è che il "male" del Peccato abbia comportato tre "beni" insperati:

Trois biens non esperez: scavoir, gloire pour soy

Vergongne pour Sathan, felicité pour toi

Three unexpected goods: praise for His name

Bliss for thyself; for Satan endless shame.

Di quei beni, osserva Lovejoy, Milton ne riprende due (lib. XII, vv. 476-477), mentre al terzo, la "vergogna di Satana", sostituisce la sovrabbondanza della grazia divina (lib. XII, v. 478). Seguono l'analisi di nove versi del poema The Triumph of Christ di Giles Fletcher il Giovane (1586-1623), che s'interroga se il Peccato fu un male o un bene, 


\section{[...] I cannot wiss}

To call it a mishap, or happy miss,

That fell from Eden... (ivi, p. 283),

e di dodici versi da L'Adamo di Giovan Battista Andreini (1576-1654) dove, in «an evident adumbration of the paradox» (ivi, p. 284), a Dio si attribuisce il potere di unire il bene e il male:

Con l'error la salute

E con l'Inferno il cielo

Insieme unir, non è potere umano,

Ma de l'eterno mano.

Nei $\$ \$ 2$ e 3 Lovejoy elenca più antiche occorrenze del paradosso: Gregorio Magno (540-604), "“O felix culpa, quae talem ac tantum meruit habere Redemptorem!”» (ivi, p. 285); Ambrogio (340-397), “"Fructuosior culpa quam innocentia” (ivi, p. 288); Leone I (390-461), «Ampliora adepti per ineffabilem Christi gratiam quam per diaboli amiseramus invidiam"» (ivi, p. 288); l'Exultet, inno liturgico del $\mathrm{V}$ secolo, nel quale il paradosso si colora della determinazione che per il maggior bene la Caduta fosse necessaria: «"O certe necessarium Adae peccatum”» (ivi, p. 285). Nei $\$ \$ 4$ e 5, dove esamina l'ulteriore ampliamento dell'idea-paradosso alla teodicea, Lovejoy passa senza soluzione di continuità da un'occorrenza di Agostino (354-430), “"Nam nisi esset hoc bonum, ut essent et mala, nullo modo sinerentur ab omnipotente bono"» (ivi, p. 290), ad occorrenze più tarde di circa un millennio: esamina brevemente John Wyclif (1320-1384), " "alle thingis comen for the beste; for alle comen for Goddis ordenance" (ivi, p. 291); quattro versi dal poema Visio Willelmi de Piers Plowman di William Landland (1332-1386), dove s'afferma che fu Dio a permettere che Adamo peccasse, "And al for the best, as I bileve” (ivi, p. 291); dieci versi da una lirica anonima del Quattrocento, che benedicono la Caduta:

Blessed be the time

That appil také was. (ivi, p. 292);

seguono un passo di Benedetto Pereira SJ (1535-1610), che loda Dio per aver mutato il Peccato in Redenzione, ed uno dal Traité de l'amour de Dieu (ivi, p. 293) di Francesco di Sales (1567-1622), sulla sovrabbondanza di Grazia provocata dal Peccato: «[...] et tant s'en faut que le peché d'Adam ait surmonté la debonnaireté divine, que tout au contraire il l'a excitée et provoqueé [...] 
elle a fait "surabonder la grace où l'iniquité avoit abondé"» (FRANCESCO DI S., 1899, p. 394). Lidea del paradosso, conclude Lovejoy, non è originale di Milton, che in Paradise Lost si limita a trattare separatamente i due corni: nella narrazione degli eventi edenici la Caduta è presentata come un male; nel XII libro, dove descrive la profezia di Michele e l'uscita dall'Eden, come un bene.

Spero che questa breve analisi basti a chiarire la differenza d'impostazione storiografica tra Lovejoy e Gerbi. Ho evidenziato le date di nascita e morte degli autori e la brevità dei testi citati, per mostrare anche intuitivamente che la ricerca di Lovejoy spazia sincronicamente attraverso i secoli, come se nel lunghissimo periodo storico che va dalla patristica a Milton l'idea del "paradosso" restasse sostanzialmente la medesima (pur nelle tre sfumature: il male è anche un bene; è condizione necessaria del bene; è giustificato dalla teodicea). Oltre alla brevità dei testi esaminati, a mantenere questa uguaglianza concorre l'astrazione dai contesti teorici e storici. Le idee di Agostino (dodici righe) e di Wyclif (otto) riguardo alla teodicea sono presentate, a distanza d'un millennio, come uguali, prescindendo dalle differenze che in esse involvono i contesti culturali, filosofici, politici ed ecclesiali. Contesti che per Agostino sono vastissimi, andando da Cicerone allo scetticismo accademico e al neoplatonismo e comprendendo inesauste polemiche col manicheismo, col pelagianesimo ecc.; meno vasti i contesti di Wyclif, ma anch'essi densi di riferimenti alla tradizione oxoniense della Scolastica, alle controversie tra corone e papato, alle istanze riformatrici e morali del movimento dei lollardi ecc.

È ben vero, per stare all'esempio di Agostino, che nemmeno Gerbi si dilunga sull'estrema complessità della sua opera. Ma ciò discende dalla necessità, in un libro di storia, di attenersi alla misura intrinseca alla ricerca, e non intacca l'impostazione volta a inserire il pensiero di Agostino nello svolgimento storico. Gerbi indica con chiarezza che fu Ambrogio, «studiosissimo di Clemente, d'Origene, e, soprattutto, di Filone», a «introdu[rre] in Occidente i metodi [allegorici] di interpretazione biblica» (p. 40), e che fu da lui che Agostino, suo "catecumeno e [...] discepolo", ereditò quel secolare dibattito, del quale divenne il «ferreo sistematore» rielaborando «profondamente l'ipotesi» carnale e deducendone con «rigore le ultime conseguenze» (p. 44). In questa prospettiva della storia come svolgimento, lungi dall'identificare idee simili, ma separate da secoli e secoli di storia, Gerbi mostra che in due autori anche vicinissimi, come Ambrogio e Agostino, l'idea del ruolo svolto nella Caduta dalla lussuria varia in relazione ai due differenti contesti di pensiero: in Ambrogio la carnalità ha tolleranti venature 
affettive, mentre in Agostino sfocia nelle cupa concezione dell'umanità massa damnata. La differenza d'impostazioni storiografica è manifesta anche nelle trattazioni di Milton: Lovejoy disegna una mappa sincronica del "paradosso" e ne trae la conclusione che Milton non sia originale; Gerbi invece colloca le "contraddittorietà" di Milton al crocevia di un lungo e complesso sviluppo storico e le collega agli aspetti contrastanti della sua personalità e all'insieme della sua opera, facendo riferimento anche all'Aereopagitica e a Samson Agonistes.

Per mostrare con un esempio conclusivo che quanto Gerbi dista dall'ispirazione fondamentale di Lovejoy, tanto è prossimo a quella di Croce, cito una tarda pagina crociana, Il peccato originale (1950), accolta nel volume della Ricciardi. Croce vi scrive che «[...] la vera storia [...] non è quella che insegue le colpe degli uomini, ma l'altra, che intende a dire ciò che l'uomo ha fatto» (CROCE, 1951, p. 679), e conclude che l'«[...] unità della vita nel bene e nel male è il vero peccato originale», che «non ha redenzione» perché la «[...] lotta del bene col male» è il nostro retaggio, senza cui non ci sarebbe legge morale (ivi, p. 678). Qui la consonanza tra Croce e Gerbi è particolarmente significativa, e Gerbi la professa quando, scomparso da pochi mesi Croce, scrive che «[...] la piena accettazione della filosofia della prassi [di Croce] implica [...] un nuovo atteggiamento verso gli uomini e verso Dio, un nuovo concetto di Peccato Originale» (GERBI, A., 1953, p. 69). È l'atteggiamento che vent'anni prima aveva espresso nelle pagine conclusive dell'Adamo ed Eva: che cademmo «[...] per farci davvero uomini. Da allora, né possiamo liberamente agire come dei, né ci possiamo acquetare nella coscienza della nostra miseria. Né bestie, né Dio. Uomini» (p. 203), emissi de paradiso voluptatis, ut consequeremur libertatem.

RAMBALDI, Enrico Isacco Crocean Historiography and the history of ideas: Antonello Gerbi’s Adamo ed Eva. Trans/Form/Ação, Marília, v. 37, p. 9-36, 2014. Edição Especial.

\footnotetext{
ABSTRACT: Antonello Gerbi (1904-1976), historian of philosophy and political thought, was very close to Benedetto Croce and in contact with Arthur Lovejoy. Following the historiographical method adopted by Croce, his book Il peccato di Adamo ed Eva [The Sin of Adam and Eve] relates the history of the conception of Original Sin as carnal sin from the II to the XIX centuries. Due to the very wide range of sources examined (philosophers, theologians, poets, artists, etc.) and the thoroughness with which he unifies his research around the central theme of the carnal nature of the Fall, the work has interesting affinities with the history of ideas and bears witness to the versatility of Croce's historiographical method, amenable to being integrated with that of Lovejoy.
}

KEYWORDS: Original Sin. Croce. Lovejoy. 


\section{FONDI ARCHIVISTICI}

ASI-BCI-P-GERBI = Archivio Storico di Intesa Sanpaolo, patrimonio Banca Commerciale Italiana (ASI-BCI), Carte personali di Antonello Gerbi (P-GERBI),

\section{Bibliografia}

Aa.Vv. Omaggio a Benedetto Croce: saggi sull'uomo e sull'opera. [Torino]: Radio Italiana, 1953.

ABBRI, Ferdinando. Paolo Rossi (1923-2012). Rivista di Storia della Filosofia, v. 69, n. 2, p. 335-353, 2014.

BERGSON, Henri. Les deux sources de la morale et de la religion (1932). In:

Euvres. Annotate da André Robinet e con introduzione di Henri Gouhier. Paris: Presses Universitaires de France, 1970. p. 979-1247.

CONTINI, Gianfranco. Altri esercizi (1942-1971). Torino: Einaudi, 1972.

CROCE, Benedetto. Filosofia della pratica: economica ed etica. Bari: Laterza, 1909.

. Prefazione. In: ERASMO DA ROTTERDAM. Elogio della pazzia e dialoghi. Traduzioni italiane di varii, coi disegni, a illustrazione dell'Elogio, di Hans Holbein. A cura di Benedetto Croce. Bari: Laterza,1914. p. v-x.

. I limiti della dottrina dello Stato come potenza. La Critica, v. 14, p. 241-242, 1916 a.

. Contro il secolo decimottavo. La Critica, v. 14, p. 243-244, 1916 b.

. Poesia e non poesia: note sulla letteratura europea del secolo decimonono. Bari: Laterza, 1923. Cito dall'ediz. $1955^{6}$.

. Rec. a Antonello Gerbi: Il peccato di Adamo ed Eva: storia della ipotesi di Beverland. La Critica, v. 21, p. 377-378, 1933.

. La storia come pensiero e come azione: anticipazione dell'omonimo volume del 1938. La Critica, v. 35, p. 1-35, 1937.

. La storia come pensiero e come azione. Bari: Laterza, 1938. Cito dall'ediz. $1954^{6}$.

. Rec. a Jean Pommier: La mystique de Marce1 Proust. (Paris: Droz, 1939). La Critica, v. 37, p. 450, 1939.

. Il peccato originale. In: CROCE, B. Filosofia, poesia, storia: pagine tratte da tutte le opere a cura dell'Autore. Milano-Napoli: Ricciardi, 1951. p. 678-680.

. Filosofia, poesia, storia: pagine tratte da tutte le opere a cura dell'Autore. MilanoNapoli: Ricciardi, 1951. (1'”Avvertenza" in cui è ringraziato Gerbi è alla c. 3r.)

. Scritti e discorsi politici (1943-1947). A cura di Angela Carella. Napoli : Bibliopolis, 1993. 
DI GIOVANNI, Piero (a cura di). Le avanguardie della filosofia italiana nel XX secolo. Milano: Angeli, 2002.

ERASMO DA ROTTERDAM. Elogio della pazzia e Dialoghi. Traduzioni italiane di varii, coi disegni, a illustrazione dell'Elogio, di Hans Holbein. A cura di Benedetto Croce. Bari: Laterza, 1914.

FRANCESCO DI SALES. Traité de l'amour de Dieu. In: . Euvres complètes. Paris: Vives, 1899. t. 1, p. 319-500.

GARIN, Eugenio. La cultura italiana tra '800 e '900. Bari: Laterza, 1962.

GERBI, Antonello. La politica del settecento: storia di un’idea. Bari: Laterza, 1928.

. Viejas polémicas sobre el Nuevo Mundo: en el umbral de una conciencia americana. Lima: Banco de Crédito, $1946^{3}$.

Appendice. In: CROCE, B. Filosofia, poesia, storia: pagine tratte da tutte le opere a cura dell'Autore. Milano-Napoli: Ricciardi, 1951. p. 1175-1237.

- La filosofia della pratica. In: Aa.Vv. Omaggio a Benedetto Croce: saggi sull'uomo e sull'opera. [Torino]: Radio Italiana, 1953. p. 65-78.

.La disputa del Nuovo Mondo: storia di una polemica (p. 1750-1900). Prima ed. Milano-Napoli: Ricciardi, 1955. - Nuova ed. a cura di Sandro Gerbi, con un "Profilo dell'autore" di Piero Treves (XIX-LXII) e la "Bibliografia degli scritti di Antonello Gerbi" a cura di Sandro Gerbi (LXIII-CVIII). Milano-Napoli: Ricciardi, 1983. - Altra nuova ed. a cura di Sandro Gerbi, con un saggio di Antonio Melis. Milano: Adelphi, 2000.

. Germania e dintorni (1929-1933). A cura di Sandro Gerbi. Milano-Napoli: Ricciardi, 1993.

. Il peccato di Adamo ed Eva: storia della ipotesi di Beverland. A cura di Sandro Gerbi. Milano: Adelphi, $2011^{2}$. Prima ed. Milano: La Cultura, 1933.

GERBI, Sandro. Raffaele Mattioli e il filosofo domato. Einaudi: Torino, 2002.

Mio padre. In: PERASSI, Emilia; PINO, Francesca (a cura di). Antonello Gerbi tra vecchio e nuovo mondo. Milano: Cisalpino-Istituto Editorial e Universitario-Monduzzi, 2009. P. 41-51. (Quaderno di Acme, Facoltà di Lettere, Università degli Studi di Milano, n. 114).

. Quarant'anni di appunti. In: GERBI, A. Il peccato di Adamo ed Eva: storia della ipotesi di Beverland. A cura di Sandro Gerbi. Milano: Adelphi, 2011. p. 237-258.

HEGEL, Giorgio Guglielmo Federico. Lezioni sulla filosofia della storia. A cura di Giovanni Bonacina e Livio Sichirollo. Bari-Roma: Laterza, 2003.

KANT, Immanuel. Congetture sull'origine della storia (1786). In: Scritti politici e di filosofia della storia e del diritto. Con un saggio di Christian Garve; tradd. di Gioele Solari e Giovanni Vidari. Edizione postuma a cura di Norberto Bobbio, Luigi Firpo e Vittorio Mathieu. Torino: Utet, $1965^{2}$. 
LOVEJOY, Arthur O. Essays in the history of ideas. Baltimore: John Hopkins Press, 1948. . La Grande Catena dell'essere. Trad. di Lia Formigari. Milano: Feltrinelli, 1966. L'albero della conoscenza: saggi di storia delle idee. Trad. di Dolores de Vera Pardini, con Introduzione di Paolo Rossi. Bologna: il Mulino, 1982.

MASUCCIO SALERNITANO. Il novellino. A cura di Alfredo Mauro. Bari: Laterza, 1940.

MELIS, Antonio . Una disputa che continua. In: GERBI, A. La disputa del Nuovo Mondo: storia di una polemica (p. 1750-1900). Altra nuova ed. a cura di Sandro Gerbi, con un saggio di Antonio Melis. Milano: Adelphi, 2000. p. 937-948.

PERASSI, Emilia; PINO, Francesca (a cura di). Antonello Gerbi tra vecchio e nuovo mondo. Milano: Cisalpino-Istituto Editorial e Universitario-Monduzzi, 2009. (Quaderno di Acme, Facoltà di Lettere, Università degli Studi di Milano, n. 114)

PINO-GUIDO MONTANARI, Francesca. Un filosofo in banca: guida alle carte di Antonello Gerbi. Con la collaborazione di Barbara Costa. Torino-Roma: Intesa San PaoloEdizioni di Storia e Letteratura, 2007.

RONCHETTI, Emanuele. Introduzione alla storia delle idee: perché non possiamo fare a meno della storia. Milano: Unicopli, 2002.

. Tra le culture: Antonello Gerbi e la storia delle idee. In: DI GIOVANNI, Piero (a cura di). Le avanguardie della filosofia italiana nel XX secolo. Milano: Angeli, 2002. p. $12-140$.

Antonello Gerbi, Lovejoy e la storia delle idee: Giacomo Leopardi e Joseph de Maistre: degenerazione e progresso. In: PERASSI, Emilia; PINO, Francesca (a cura di). Antonello Gerbi tra vecchio e nuovo mondo. Milano: Cisalpino-Istituto Editorial e Universitario-Monduzzi, 2009. p. 139-163. (Quaderno di Acme, Facoltà di Lettere, Università degli Studi di Milano, n. 114).

ROSSI, Paolo. Storia e filosofia: saggi sulla storiografia filosofica. Torino: Einaudi, 1969.

Introduzione. In: LOVEJOY, Arthur O. L'albero della conoscenza: saggi di storia delle idee. Trad. di Dolores de Vera Pardini, con Introduzione di Paolo Rossi. Bologna: il Mulino, 1982. p. 7-17.

ROUSSEAU, Jean-Jacques. Émile ou de l'éducation. In: Euvres complètes. Edizione diretta da Bernard Gagnebin e Marcel Raymond, testo a cura di Chares Wirz. Paris: Gallimard, 1969. p. 239-868.

TREVES, Piero. Profilo di Antonello Gerbi. In: GERBI, A. La disputa del Nuovo Mondo: storia di una polemica (p. 1750-1900). Nuova ed. a cura di Sandro Gerbi. Milano-Napoli: Ricciardi, 1983. p. xxxix-xlii. 